\title{
Aplicação dos sistemas adesivos nos últimos cinco anos
}

\author{
Application of adhesive systems in the last five years \\ Aplicación de sistemas adesivos en los últimos cinco años \\ Paulo Ladislau SAVICZKI ${ }^{1}$ \\ Maryanne de Mendonça e Silva REBOUÇAS ${ }^{\mathbf{1}}$ \\ Christiane Toriy GENARO ${ }^{1}$ \\ Danilo Chizzolini MASOCATTO ${ }^{1}$ \\ Túlio Marcos Kalife COELHO ${ }^{2}$ \\ José Luiz Guimarães de FIGUEIREDO ${ }^{3}$ \\ Benícia Carolina Iaskieviscz RIBEIRO ${ }^{3}$ \\ ${ }^{1}$ Mestrando em Odontologia pela Universidade Federal do Mato Grosso do Sul, 79070-900 Campo Grande - MS, Brasil \\ ${ }^{2}$ Professor Adjunto do Curso de Odontologia da Universidade Federal do Mato Grosso do Sul, \\ 79070-900 Campo Grande - MS, Brasil \\ ${ }^{3}$ Professor Associado do Curso de Odontologia da Universidade Federal do Mato Grosso do Sul, \\ 79070-900 Campo Grande - MS, Brasil
}

\begin{abstract}
Resumo
Os novos materiais restauradores têm sido desenvolvidos para atender as exigências dos clínicos e obter um nível de excelência tanto em termos funcionais quanto em estética. Com isso, uma grande variedade de produtos odontológicos é lançada no mercado a cada ano, dentre esses produtos temos os sistemas adesivos odontológicos e junto a eles surgem diferentes técnicas de utilização. Portanto é estritamente importante o conhecimento do profissional durante a seleção e aplicação desses sistemas adesivos. A elaboração dessa revisão foi impulsionada devido a grande quantidade de sistemas disponíveis hoje e buscou compreender a fundo a aplicação desses sistemas de acordo com as pesquisas dos últimos 5 anos. Essas pesquisas nos trouxeram dados altamente significativos, tais como: que o aumento do tempo de aplicação dos sistemas autocondicionantes leva a uma melhora na resistência adesiva; os novos conceitos de hibridização a partir da desproteinização da dentina e fixação da clorexidina como inibidor efetivo de metaloproteinases.
\end{abstract}

Descritores: Adesivos Dentinários; Resistência Adesiva; Resinas Compostas.

\begin{abstract}
The new restorative materials have been developed to meet the requirements of clinicians and achieve a level of excellence in both functional and aesthetic terms. With this, a great variety of dental products is launched on the market every year, among these products we have the dental adhesive systems and with them come different techniques of use. Therefore, the professional's knowledge during the selection and application of these adhesive systems is strictly important. The elaboration of this review was boosted due to the large number of systems available today and sought to thoroughly understand the application of these systems according to the surveys of the last 5 years. These researches have brought us highly significant data, such as: that the increase of the time of application of the self-conditioning systems leads to an improvement in the adhesive resistance; the new concepts of hybridization from dentin deproteinization and fixation of chlorhexidine as an effective inhibitor of metalloproteinases.

Descriptors: Dentin-Bonding Agents; Tensile Strength; Composite Resins.
\end{abstract}

\section{Resumen}

Los nuevos materiales restauradores se han desarrollado para satisfacer las exigencias de los clínicos y obtener un nivel de excelencia tanto en términos funcionales como en estética. Con eso, una gran variedad de productos odontológicos es lanzada en el mercado cada año, entre estos productos tenemos los sistemas adhesivos odontológicos y junto a ellos surgen diferentes técnicas de utilización. Por lo tanto es estrictamente importante el conocimiento del profesional durante la selección y aplicación de estos sistemas adhesivos. La elaboración de esta revisión fue impulsada debido a la gran cantidad de sistemas disponibles hoy y buscó comprender a fondo la aplicación de esos sistemas de acuerdo con las investigaciones de los últimos 5 años. Estas investigaciones nos trajeron datos altamente significativos, tales como: que el aumento del tiempo de aplicación de los sistemas auto condicionantes lleva a una mejora en la resistencia adhesiva; los nuevos conceptos de hibridación a partir de la desproteinización de la dentina y la fijación de la clorhexidina como inhibidor efectivo de metaloproteinasas.

Descriptores: Recubrimientos Dentinarios; Resistencia a la Tracción; Resinas Compuestas.

\section{INTRODUÇÃO}

O surgimento dos sistemas adesivos fez com que o sucesso clínico de uma restauração dependesse do selamento marginal entre as margens do material restaurador e da cavidade. Assim, um sistema adesivo que proporcione boa resistência adesiva e longevidade clínica é essencial para o sucesso e longevidade das restaurações estéticas diretas em Resina Composta. Entretanto, alguns problemas clínicos enfrentados como a contração de polimerização e a tensão na interface resina-dentina podem conduzir à formação de espaços que geram microinfiltração marginal, descoloração marginal, cáries recorrentes, dor e sensibilidade pós-operatória ${ }^{1}$.

Dúvidas quanto ao protocolo de utilização e melhor indicação para cada sistema são comuns frente a enorme quantidade de marcas e de produtos de sistemas Adesivos disponíveis no mercado. Sabendo-se que a adesão e a força de adesão de diferentes agentes adesivos são influenciadas por inúmeros fatores (como saliva, fluido gengival, sangue, método de preparação da superfície da dentina e composição de materiais adesivos), os atuais estudos buscam verificar e avaliar a influência desses fatores na resistência à tração de sistemas adesivos de geração diferentes. O estudo de Gupta et al. ${ }^{2}$ em 2015, por exemplo, constatou que o sistema adesivo menos influenciado pela contaminação salivar era o de um passo autocondicionante, seguida pelo de dois passos autocondicionante e do dois passos etch-and-rinse respectivamente. Ainda assim, diferentes abordagens têm sido propostas para melhorar a resistência adesiva e a infiltração de monômeros em dentina desmineralizada, sendo a abordagem mais frequentemente descrita na literatura, a utilização do digluconato de clorexidina $^{2}$. 
Sabe-se que o parâmetro chave para a avaliação da qualidade dos diferentes sistemas de adesivos dentinários é a resistência de união, assim os métodos de avaliação de laboratório precisos e unificados são cruciais para a aplicação clínica dos diferentes sistemas adesivos. Porém, devido ao uso de numerosas técnicas de teste e parâmetros torna-se difícil obter dados consistentes de resistência de união ${ }^{3}$.

O objetivo deste artigo foi verificar quais estudos trabalharam diretamente com a utilização de sistemas adesivos nos últimos cinco anos e quais resultados obtiveram, sendo utilizadas as bases de dados Pubmed e Scielo. Os artigos relacionados nessa pesquisa foram selecionados a partir dos termos "adhesive system dentin application", sendo encontrados nos últimos 5 anos, 54 artigos que tratavam diretamente sobre o assunto.

\section{REVISÃO DA LITERATURA}

Em 2012, Assad-Loss et al. ${ }^{4}$ verificaram a influência da saliva na ação da resistência adesiva dos adesivos em esmalte dental. Foram avaliados 3 adesivos diferentes, sendo eles o Transbond XT, Transbond MIP e o Single Bond e utilizaram dentes bovinos com e sem contaminação de saliva para o teste de resistência adesiva, chegando a conclusão que a queda da resistência adesiva é significativa.

Mena-Serrano et al. ${ }^{5}$ verificaram a influência do tempo de aplicação do ácido fosfórico e de adesivos autocondicionantes em dentina esclerosada, verificando assim a resistência adesiva de restaurações de resina composta após os testes, foram utilizados um sistema adesivo convencional, single bond $2(3 \mathrm{M})$ e dois sistemas adesivos autocondicionantes, o sistema Scothbond SE(3M) e o sistema GO(SDI), sendo testados de acordo com as normas do fabricante e também com o dobro do tempo de condicionamento, como resultado foi observado que duplicar o tempo de condicionamento para o sistema de passo único o torna mais efetivo para o sistema testado.

Firoozmand et al. ${ }^{6}$ verificaram se a aplicação de flúor profissional interferiria na resistência adesiva de sistemas adesivos de dois passos e de passo único, os pesquisadores chegaram ao resultado que quando existe uma aplicação prévia de flúor antes da restauração é gerado um decréscimo na resistência adesiva das restaurações. Isso também foi observado em tipos diferentes de dentes.

Carvalho et al. $^{7}$ verificaram a influência do aquecimento e da temperatura do adesivo na resistência de união de um sistema adesivo à base de etano, sendo baseado na afirmação de que o aumento da temperatura do adesivo auxilia na liberação do solvente, reduzindo a viscosidade e auxiliando na penetração dos monômeros na dentina. Foram elaborados 3 grupos, sendo o primeiro o grupo controle, o segundo grupo foi conseguido por meio de um dispositivo de aquecimento, até a temperatura de 37 graus e o terceiro usou- se um jato de ar quente para esquentar as amostras para a preparação dos corpos de prova. Como resultado, os autores observaram que não houve diferença significativa entre os grupos, não importando o aquecimento do adesivo para um sistema adesivo de dois passos.

Em 2012, Dalkilic et al. ${ }^{8}$ verificaram o efeito de diferentes métodos desinfetantes na microtensão inicial de sistemas adesivos autocondicionantes. Como método de infecção foram testados 4 meios, formando-se 4 grupos, sendo que no primeiro o grupo foi utilizado gás ozônio, o segundo utilizou-se do laser $\mathrm{Nd}$ :Yag, o terceiro utilizou a Clorexidina a $2 \%$ como agente de desinfecção, o quarto foi o grupo controle. Como resultados, o menor resultado de resistência adesiva foi atribuído ao grupo OZ, sendo associado á perda de resistência adesiva, enquanto os outros grupos não geraram alterações significativas na resistência adesiva.

Caneppele et al. ${ }^{9}$ verificaram os efeitos da hidratação superficial e o método de aplicação dos sistemas adesivos na resistência adesiva dos sistemas auto condicionantes. Foram avaliados 195 dentes bovinos, 15 considerados como parâmetro de controle e aplicado o sistema adesivo single bond 2, de acordo com a normas do fabricante. Os demais dentes foram separados em três grupos de acordo com sistema adesivo utilizado, sendo utilizados o Futurabond $\mathrm{M}$ (Voco), Clearfill S $\mathrm{S}^{3}$ Bond (Kuraray) e o adesivo Optibond (kerr). Em relação à aplicação do adesivo foram utilizados dois métodos, passivo, sendo aplicado sem esfregaço e ativo, com esfregaço de ponta de aplicação, foi também utilizado dois meios de controle de umidade, a secagem por ar e por papel absorvente. $\mathrm{O}$ adesivo que provou uma melhor resistência adesiva foi Futurabond M (Voco) e a aplicação ativa do adesivo foi a mais eficaz significativamente, não importando o meio de umidade.

Em 2012, Unlu et al. ${ }^{10}$ observaram a influência da experiência do operador na resistência adesiva dos sistemas adesivos in vitro. Foi analisado o adesivo autocondicionante (Clearfill SE Bond, Kuraray) e o adesivo de dois passos (Single Bond, 3m espe) em 120 terceiros molares humanos preparados para os testes. Foram selecionados 4 grupos de profissionais, em diferentes níveis, sendo um especialista em dentística, um estudante da pós graduação, um estudante graduando e um clínico generalista. Como resultado os pesquisadores chegaram a conclusão de que a experiência é de grande valia para os procedimentos operatórios, sendo significante para a condição adesiva. Nesse estudo, quanto maior a graduação dos profissionais, maiores foram os resultados de resistência adesiva.

Também em 2012 Khoroushi e Saneie ${ }^{11}$ avaliaram o efeito dos antioxidantes usados após clareamento em relação à resistência adesiva de três sistemas adesivos. Foi avaliado um grupo controle negativo, um grupo controle negativo, um grupo com espera na aplicação do adesivo e um grupo com ascorbato de sódio como agente antioxidante. Chegou-se a conclusão que o ascorbato de sódio é efetivo no aumento da resistência adesiva quando comparado ao grupo controle positivo e também aumenta a resistência adesiva dos adesivos autocondicionantes. Também verificaram que o delay durante a aplicação do adesivo nesse estudo não alterou a resistência adesiva dos adesivos autocondicionantes e concluíram que os adesivos de dois passos são mais efetivos no aumento da resistência adesiva de forma geral.

Cantanhede de Sá et al. ${ }^{12}$ analisaram os efeitos do armazenamento em água de corpos de prova, na resistência adesiva e na habilidade de selamento dos sistemas adesivos na dentina, sendo avaliados em 24 horas e seis meses após armazenamento. Foram utilizados dois grupos de sistemas adesivos, um de condicionamento e lavagem e um de passo único. Os autores concluíram que não há mudança em relação á resistência adesiva, porém os adesivos autocondicionantes mostraram um melhor selamento da dentina após seis meses, mas todos os sistemas apresentaram uma diminuição do selamento quando armazenados em água.

Lenzi et al. ${ }^{13}$ pesquisaram a influência da clorexidina 
no aumento da resistência adesiva na utilização de sistemas de três passos, em dentes permanentes com dentina afetada. Verificaram e confirmaram que a utilização da clorexidina não interfere na resistência adesiva quando aplicada em dentes com dentina afetada.

Sauro et al. ${ }^{14}$ verificaram a resistência adesiva de um sistema adesivo autocondicionante em preparos condicionados a jateamento abrasivo com vidro bioativo e ácido poliacrilico, os autores verificaram que essa tentativa de se aumentar a resistência adesiva não obteve sucesso.

Os efeitos da saliva e sangue na resistência adesiva de sistemas adesivos autocondicionantes foram estudados por Koppolu et al. ${ }^{15}$. Como esperado, o estudo chegou a conclusão que saliva e sangue diminuem significativamente a resistência adesiva dos sistemas adesivos.

Atabek et al. ${ }^{16}$ verificaram a resistência adesiva dos sistemas adesivos em relação ao MTA, em diferentes intervalos de tempo. 20 cilindros de acrílico foram confeccionados e aplicados MTA e separados em 5 grupos, 40 unidades foram armazenadas por 4, 24, 48, 72 e 96 horas, por 37 graus e $100 \%$ de umidade. Nesses grupos foram aplicados os sistemas adesivos e levados á maquina de ensaios universais, como resultado, foi verificado uma melhor condição adesiva após 72 horas, sendo indicado procedimentos restauradores após 96 horas.

Carvalho et al. ${ }^{17}$ analisaram o efeito dos sistemas adesivos e a estratégia de aplicação na redução da permeabilidade da dentina. Nenhum dos adesivos analisados chegou á $100 \%$ de selamento em dentina bovina, entretanto todos os adesivos aplicados geraram um selamento próximo a $90 \%$.

A resistência adesiva de sistemas adesivos autocondicionantes em diferentes condições de umidade e secagem com ar foi estudada por Lee et al. ${ }^{18}$, sendo a condição de aplicação de ar em dentina seca, após aplicação do sistema adesivo, a que se mostrou a mais efetiva nesse estudo.

Jia et al. ${ }^{19}$ analisaram a influência da contaminação de recidiva de cárie na resistência adesiva de diferentes adesivos na dentina. $\mathrm{O}$ estudo foi simulado por meio do sistema Caries Infiltrant System (Icon DMG). Foi simulada a infiltração de cárie, com ácido clorídrico a $15 \%$ e uma solução de resina infiltrante, associados a um grupo controle e aplicaram-se três sistemas adesivos diferentes. O trabalho concluiu que a ação da cárie sozinha, não possui efeito em relação ao desempenho do sistema adesivo, porém quando existe a contaminação por HCL, existe uma interferência direta na resistência adesiva.

Pupo et al. ${ }^{20}$ verificaram a influência da variabilidade da dentina e o modo de aplicação dos sistemas adesivos nas propriedades mecânicas da camada adesiva. O estudo verificou a dificuldade de se analisar diferentes tipos de dentina para se formar um padrão e concluiu que a resistência adesiva é dependente diretamente da profundidade do preparo e da forma de aplicação.

Em 2013 Sartori et al. ${ }^{21}$ avaliaram a efetividade de uma camada hidrofóbica de resina, utilizada associada a um adesivo de passo único. O acompanhamento após 18 meses desse trabalho verificou que não aconteceu um aumento de resistência adesiva dessas restaurações em lesões não cariosas no período observado.

No mesmo ano Marimoto et al. ${ }^{22}$ verificaram a influência do laser Nd:YAG, na resistência adesiva de sistemas adesivos convencionais e autocondicionantes e também sua ação sobre os tecidos dentários. Nesse estudo o adesivo autocondicionante apresentou um maior valor de resistência adesiva, quando comparado a um sistema convencional. Foi verificado também que a polimerização do sistema adesivo e o uso do laser Nd:YAG geraram um aumento na resistência adesiva.

Também em 2013 Arslan et al. ${ }^{23}$ verificaram o efeito de contaminação de um agente hemostático e sangue em microfraturas de sistemas adesivos de um e dois passos. Os autores concluíram que a contaminação com sangue previamente a aplicação dos adesivos aumentam o índice de microfraturas. Foi verificado que o agente hemostático em si não altera a resistência adesiva.

Nowicka et al. ${ }^{24}$ verificaram a resposta pulpar de dentes após capeamento com adesivos autocondicionantes. Verificou-se que houve uma organização para formação de ponte dentinária nos dentes que foram aplicados os adesivos autocondicionantes, porém, nenhum obteve a efetividade do hidróxido de cálcio em todos os aspectos observados.

Batra et al. ${ }^{25}$ verificaram, em 2013 a resistência adesiva de três diferentes sistemas adesivos de três passos após condicionamento ácido adicional com ácido fosfórico. Nesse estudo foi utilizado os sistemas Clearfill $\mathrm{S}^{3}$ Bond, Futurabond NR e Xeno V e como grupo controle foi utilizado o sistema Single Bond 2. Como resultado foi verificado que o condicionamento ácido em esmalte aumenta de forma efetiva a resistência adesiva nas aplicações dos adesivos autocondicionantes.

Pei et al. ${ }^{26}$ avaliaram o efeito do pré tratamento com dessensibilizante a base de cálcio na adesão á dentina de sistemas adesivos autocondicionantes. Foram utilizados dois sistemas adesivos, G-bond e o Clearfill $\mathrm{S}^{3}$ e utilizados 4 grupos: controle, grupo que utilizou a pasta sensitive pro relief, pasta de dentes e pasta de hidroxiapatita,. Como resultado não houve diferença significativa no valor de resistência adesiva dos sistemas adesivos apresentados e houve uma indicação de aplicação de pastas a base de hidróxido de cálcio para prevenção de sensibilidade.

Simões et al. ${ }^{27}$ analisaram a influência do etanol e clorexidina na resistência adesiva dos sistemas autocondicionantes. Os modelos de pesquisa foram armazenados 24 horas e seis meses e chegaram a conclusão que a mistura de clorexidina associada ao etanol, não é capaz de degenerar a camada adesiva, não alterando a condição de resistência adesiva.

Mena-Serrano et al. $^{5}$ verificaram a influência do tempo de aplicação do ácido fosfórico e de adesivos autocondicionantes em dentina esclerótica. Verificaram que a duplicação do tempo de aplicação tem influência direta na resistência adesiva de adesivos autocondicionantes.

Um novo modelo de preparo de dentina, utilizando enzimas para desproteinizar a dentina, a fim de uma melhora nas propriedades adesivas da mesma foi estudado por Dayem et al. ${ }^{28}$. Como resultado obtido, verificaram que a utilização da enzima para desproteinizar a dentina por um minuto é suficiente para a redução do colágeno e diminuição do índice de microfraturas na superfície adesiva.

Perdigão et al. $^{29}$ em 2014, verificaram as propriedades adesivas imediatas em dentina e em esmalte do adesivo universal associado a uma camada hidrofóbica de resina. Os autores observaram aumento da resistência adesiva dos adesivos, tanto do autocondicionante quanto do convencional. Concluíram que o uso de uma camada de resina hidrofóbica associado ao sistema adesivo pode ser uma ferramenta de auxílio no uso de adesivos autocondicionantes.

Em 2014 Malekipour et al. ${ }^{30}$ avaliaram a resposta da polpa humana aos sistemas adesivos convencionais e 
autocondicionantes. O experimento clínico foi realizado em pré molares dos voluntários, sendo aplicando os adesivos Single Bond e o Prompt L-Pop e depois de 30 dias os dentes foram extraídos e avaliados. Observou-se uma infusão de células inflamatórias e formação de ponte de dentina levando a resolução de que esses sistemas adesivos apresentam boa compatibilidade biológica.

No mesmo ano Thanatvarakorn et al. ${ }^{31}$ observaram o efeito eliminador de proteína do hipoclorito de sódio e do ácido hipocloroso no pré-tratamento da smear layer e seus efeitos nas características da interface da dentina-resina de sistemas adesivos autocondicionantes. Concluíram que o pré-tratamento da dentina com essas soluções leva a um preparo superficial, podendo eliminar a camada híbrida, levando a uma redução da espessura da camada de união adesiva e a uma redução da nano infiltração.

Também em 2014 Marchesi et al. ${ }^{32}$ avaliaram a estabilidade adesiva de um adesivo de passo único, utilizando diferentes técnicas adesivas, em dentina humana. 60 terceiros molares humanos íntegros foram preparados e separados em 4 grupos: Scothbond Universal self etch, Scothbond Universal ER com meio úmido, Scothbond Universal ER com meio seco, Prime e Bond NT, em tempo zero de estocagem, seis meses e um ano. Os resultados suportam que o uso de adesivos autocondicionantes manteve a estabilidade o ano todo.

Muñoz et al. ${ }^{33}$ em 2014, assim como Perdigão et al. ${ }^{30}$ em 2013 realizaram um estudo sobre a utilização de uma aplicação de resina hidrofóbica associada á utilização de três adesivos universais. A conclusão leva a constatação que essa associação aumenta o desempenho da união dentinaresina, mas é dependente da composição do sistema adesivo utilizado.

Loguercio et al. ${ }^{34}$ realizaram a avaliação laboratorial dos adesivos de três passos, avaliando de diversos modos o funcionamento dos adesivos citados. Os mesmos foram filetados para avaliação na máquina de ensaios universais e imersos em uma solução de prata, além disso, foi avaliado o grau de conversão in situ e microfraturas. Como resultado do trabalho, todos os sistemas adesivos apresentaram excelente desempenho laboratorial, sendo o melhor nesse trabalho o ScothBond Multi-Purpose.

A possibilidade dos adesivos autocondicionantes terem uma relação com as microfraturas de restaurações classe V foi avaliada por Mirmohammadiet al. ${ }^{35}$ em 2014. Esse estudo não encontrou relação entre o adesivo e a situação de microfraturas, sendo indicados estudos de maior espaço de tempo para representação do mesmo.

Wagner et al. $^{36}$ estudaram a performance dos adesivos universais em diferentes métodos de condicionamento ácido utilizando o modo autocondicionante e o modo de condicionamento e lavagem, submetendo os grupos à termociclagem. Concluiu então que o pré-condicionamento do substrato dental provê uma maior relação adesivo/substrato, gerando maiores tags na camada adesiva, porém, isso não interfere diretamente na resistência adesiva e que a condição da termociclagem gera a degradação da camada adesiva, levando a uma queda da resistência adesiva de forma direta.

Em 2014 também Rodríguez-Priego et al. ${ }^{37}$ avaliaram a possibilidade de alteração na condição adesiva da dentina primária de pessoas que ingerem sulfato ferroso. Os pesquisadores concluíram que o sulfato ferroso, altera a microquímica estrutural da dentina primária, impactando na resistência adesiva de sistemas adesivos autocondicionantes, não se aplicando aos convencionais.
Elkassas et al. ${ }^{38}$ avaliaram os efeitos de agentes de limpeza cavitário na resistência adesiva. Foram utilizados 4 agentes de limpeza cavitário: hipoclorito de sódio $5.25 \%$, Clorexidina, Tubulicid red e biopure, sendo aplicados sistemas adesivos autocondicionantes e convencionais. No grupo dos adesivos autocondicionantes, não houve diferença de resistência adesiva em relação ao Clearfill $\mathrm{S}^{3}$, sendo sua resistência adesiva comparada ao adesivo condicional Single Bond, enquanto para os adesivos convencionais, os agentes de limpeza não alteraram a resistência adesiva.

E ainda em 2014, Moghaddas et al. ${ }^{39}$ avaliaram as microfraturas dos sistemas adesivos autocondicionantes e convencionais, seguido de irrigação com hipoclorito de sódio. Como conclusão verificou-se que nos dois sistemas adesivos o índice de microfraturas é similar.

Aman et al. ${ }^{40}$ em 2015 realizaram um estudo que verificou a retenção dos selantes em sistemas adesivos autocondicionantes e convencionais. Como conclusão desse estudo verificou-se que os selantes empregados com sistemas adesivos convencionais apresentaram um alto índice de sucesso quando comparado com os autocondicionantes.

Almahdy et al. $^{41}$ analisaram o funcionamento da adição de inibidor de metaloproteinases à matriz do primer do sistema adesivo de três passos e verificaram sua interferência na interface adesiva em dentina cariada. Como resultados desse estudo observou-se que a adição de inibidor de metaloproteinases no primer adesivo não alterou a interface adesiva durante o período do estudo.

Vinagre et al. $^{42}$ em 2015 analisaram a resistência adesiva e a micromorfologia de dentes cortados com ponta diamantada utilizando 5 sistemas adesivos. Nesse estudo chegaram a conclusão que os maiores valores de resistência adesiva foram encontrados nos sistemas adesivos Optibond FL, Prime e Bond NT, Clearfill SE Bond, sendo o pior resultado no sistema Xeno V+.

Tranquilin et al. ${ }^{43}$ verificaram a inclusão do $\mathrm{TiF}^{4}$, no pré tratamento dentinário a fim de aumentar a resistência adesiva nos sistemas adesivos de dois passos. Como resultados obtiveram um aumento na resistência adesiva na inclusão desse composto.

Amsler et al. ${ }^{44}$ em 2015, verificaram a resistência adesiva das restaurações em dentina, com diferentes sistemas adesivos, com a influência de relativa umidade e tempo de aplicação. Os autores que o aumento da umidade age de forma a diminuir a resistência adesiva, mas uma redução do tempo de aplicação do adesivo não gera efeito.

Mithiborwala et al. ${ }^{45}$ avaliaram a eficácia do condicionamento total e auto condicionamento dentinário em dentes decíduos. Chegaram à conclusão que o sistema adesivo Prime e Bond NT, gerou um padrão de condicionamento dentário parecido com o de Silverstone do tipo II, provendo uma adesão mais adequada e uma maior longevidade à restauração.

Alves e Sobral $^{46}$ em 2015 analisaram a biocompatibilidade dos sistemas adesivos autocondicionantes baseados em butanol terciário, aplicado em cavidades profundas de dentes humanos nos intervalos de tempo de 01, 07, 14 e 21 dias. O estudo apresentou como resultado uma situação satisfatória em relação à compatibilidade.

Sezinando et al. $^{47}$ também em 2015 verificaram a utilização de uma camada de resina hidrofóbica imediatamente e seis meses após, da adesão dentinária em três adesivos universais. Como nos outros estudos posteriormente citados essa situação só foi efetiva com os 
adesivos autocondicionantes.

No mesmo ano Sharafeddin e Farhadpour $^{48}$ avaliaram a resistência adesiva de sistemas adesivos convencionais e autocondicionantes, após aplicação de clorexidina em dentina contaminada e com agente hemostático. A contaminação do agente hemostático na dentina reduziu efetivamente a resistência adesiva e quando analisado somente a clorexidina isolada não teve-se alteração na resistência adesiva.

Gupta et al. ${ }^{2}$ em 2015 avaliaram o efeito da saliva em relação á resistência adesiva de diferentes sistemas adesivos e chegaram a conclusão que o sistema adesivo que menos sofre influência da saliva é o sistema autocondicionante de um passo, seguido do sistema de dois passos autocondicionante e o de dois passos convencional, respectivamente.

$\mathrm{O}$ efeito desproteinizador da enzima bromelain na resistência adesiva foi estudado por Chauhan et al. ${ }^{49}$. Como resultado os autores verificaram que os dentes condicionados pela enzima obtiveram um aumento significante na resistência adesiva.

Montagner et al. $^{50}$ avaliaram o efeito do prétratamento dentinário com hipoclorito de sódio e sua ação na resistência adesiva. Como resultado observaram que a ação de desproteinizar a dentina na pré-restauração não interfere diretamente na resistência adesiva dos sistemas adesivos.

Ainda em 2015 Silva et al. ${ }^{1}$ analisaram a influência da clorexidina na adesão dentinária de dois sistemas adesivos depois de armazenamento em água destilada, óleo mineral e hipoclorito de sódio a $1 \%$, em 24 horas e 15 dias. Como resultado a clorexidina não apresentou alteração na resistência adesiva nas situações avaliadas, porém a clorexidina influencia negativamente a resistência adesiva do Single Bond 2 em 15 dias de armazenamento em hipoclorito a $1 \%$.

Carvalho et al. ${ }^{51}$ estudaram em 2016, os efeitos do extrato de chá verde, na durabilidade da adesão de adesivos autocondicionantes, em dentina afetada. Seu estudo concluiu que a aplicação do extrato de chá verde como inibidor de metaloproteinases age de forma favorável a adesão. O estudo também concluiu que houve uma perda de resistência adesiva quando clorexidina é utilizada e armazenada em água.

No mesmo ano Takada et al. ${ }^{52}$ observaram o efeito da aplicação de sistemas adesivos em cavidades preparadas com erbium, chromium: yttrium scandium gallium garnet laser, em 24 horas e 14 dias, como resultado os pesquisadores relataram que o preparo das cavidades com ácido fosfórico, laser ou hipoclorito de sódio não afetam o reparo pulpar de rato.

\section{CONSIDERACÕES FINAIS}

Os trabalhos relacionados aos sistemas adesivos, nos últimos 5 anos, nos mostram que o foco das pesquisas está centrado na busca pelo aumento da resistência adesiva dos adesivos autocondicionantes e os fatores que interferem nessa adesão. Dentre eles, pode-se citar o modo de aplicação desses adesivos, bem como o tempo de aplicação, os quais são fatores que podem alterar a sua resistência adesiva.

Os estudos apontaram que a possibilidade de contaminação da interface adesiva por saliva ou sangue gera um prejuízo à resistência adesiva, situação não aplicável a um agente hemostático de forma isolada.
Em termos de selamento, em situação de estocagem em água, verificou-se que os agentes autocondicionantes possuíam maior selamento em até seis meses.

Analisou-se a ação de inibidores de metaloproteinases associados aos adesivos e ainda buscamse compostos alternativos para essa função que se possa associar aos sistemas adesivos sem alterar os padrões de resistência adesiva.

Nesse período também foram realizados estudos que buscaram verificar a biocompatibilidade desses adesivos quando expostos á cavidades profundas, sendo eles razoavelmente compatíveis, mas ainda deixando a desejar quando analisamos a sua capacidade de indução de dentina.

Por fim, sugere-se uma nova percepção sobre camada adesiva, estudos tem verificado a possibilidade de se desproteinizar a camada superficial de dentina com produtos como o hipoclorito de sódio a fim de minimizar microfraturas, assim aumentando a qualidade da condição adesiva.

\section{REFERÊNCIAS}

1. Silva EM, Glir DH, Gill AWMC, Giovanini AF, Furuse AY, Gonzaga CC. Effect of chlorhexidine on dentin bond strength of two adhesive systems after storage in different media. Braz Dent J. 2015, 26(6): 642-7.

2. Gupta N, Tripathi AM, Saha S, Dhinsa K, Garg A. Effect of saliva on the tensile bond strength of different generation adhesive systems: an in-vitro study. J Clin Diagn Res. 2015; 9(7): ZC91-4.

3. Chaia Y, Lin H, Zheng G, Zhang X, Niu G, Du Q. Evaluation of the micro-shear bond strength of four adhesive systems to dentin with and without adhesive área limitation. Biomed Mater Eng. 2015; 26(Suppl 1):S63-72.

4. Assad-Loss TF, Tostes M, Mucha JN. Influence of saliva contamination on the shear bond strength of adhesives on enamel. Dental Press J Orthod. 2012; 17(2):30.e1-30.e6.

5. Mena-Serrano AP, Garcia EJ, Perez MM, Martins GC, Grande RH, Loguercio AD et al. Effect of the application time of phosphoric acid and self-etch adhesive systems to sclerotic dentin. J Appl Oral Sci. 2013; 21(2):196-202.

6. Firoozmand LM, Noleto LE, Gomes IA, Bauer JR, Ferreira MC. Effect of fluoride and simplified adhesive systems on the bond strength of primary molars and incisors. Braz Dent J. 2015, 26(4):368-73.

7. Carvalho MPM, Rocha RO, Krejci I, Bortolotto T, Bisogno FE, Susin AH. Influence of a heating device and adhesive temperature on bond strength of a simplified ethanol-based adhesive system. Rev Odontol UNESP. 2016; 45(2):97-102 .

8. Dalkilic EE, Arisu HD, Kivanc BH, Uctasli MB, Omurlu H. Effect of different disinfectant methods on the initial microtensile bond strength of a self-etch adhesive to dentin. Lasers Med Sci. 2012; 27(4):819-25.

9. Caneppele TM, Torres CR, Sassaki A, Valdetaro F, Fernandes RS, Prieto de Freitas $\mathrm{C}$ et al. Effects of surface hydration state and application method on the bond strength of self-etching adhesives to cut enamel. J Adhes Dent. 2012;14(1):25-30.

10. Unlu N, Gunal S, Ulker M, Ozer F, Blatz MB. Influence of operator experience on in vitro bond strength of dentin adhesives. J Adhes Dent. 2012;14(3):223-7.

11. Khoroushi M, Saneie T. Post-bleaching application of 
an antioxidant on dentin bond strength of three dental adhesives. Dent Res J (Isfahan). 2012; 9(1):46-53.

12. Cantanhede de Sá RB, Oliveira Carvalho A, PuppinRontani RM, Ambrosano GM, Nikaido T, Tagami J et al. Effects of water storage on bond strength and dentin sealing ability promoted by adhesive systems. J Adhes Dent. 2012; 14(6):543-9.

13. Lenzi TL, Tedesco TK, Soares FZ, Loguercio AD, Rocha Rde O. Chlorhexidine does not increase immediate bond strength of etch-and-rinse adhesive to caries-affected dentin of primary and permanent teeth. Braz Dent J. 2012; 23(4):438-42.

14. Sauro S, Watson TE, Thompson I, Banerjee A. Onebottle self-etching adhesives applied to dentine airabraded using bioactive glasses containing polyacrylic acid: An in vitro microtensile bond strength and confocal microscopy study. J Dent. 2012; 40(11):896-905.

15. Koppolu M, Gogala D, Mathew VB, Thangala V, Deepthi M, Sasidhar N. Effect of saliva and blood contamination on the bond strength of self-etching adhesive system- An in vitro study. J Conserv Dent. 2012; 15(3):270-3.

16. Atabek D, Sillelioğlu H, Olmez A. Bond strength of adhesive systems to mineral trioxide aggregate with different time intervals. J Endod. 2012; 38(9):1288-92.

17. Carvalho AO, Oliveira MT, Nikaido T, Tagami J, Gianinni M. Effect of adhesive system and application strategy on reduction of dentin permeability. Braz Oral Res. 2012; 26(5):397-403.

18. Lee Y, Park JW. Effect of moisture and drying time on the bond strength of the one-step self-etching adhesive system. Restor Dent Endod. 2012; 37(3):155-9.

19. Jia L, Stawarczyk B, Schmidlin PR, Attin T, Wiegand A. Influence of caries infiltrant contamination on shear bond strength of different adhesives to dentin. Clin Oral Investig. 2013;17(2):643-8.

20. Pupo YM, Michél MD, Gomes OMM, Lepienski CM, Gomes JC. Effect of the regional variability of dentinal substrate and modes of application of adhesive systems on the mechanical properties of the adhesive layer. $\mathrm{J}$ Conserv Dent. 2012; 15(2):132-6.

21. Sartori N, Peruchi LD, Guimarães JC, Silva SB, Monteiro S Jr, Baratieri LN, Belli R. Clinical effectiveness of a hydrophobic coating used in conjunction with a one-step self-etch adhesive: an 18month evaluation. Oper Dent. 2013; 38(3):249-57.

22. Marimoto AK, Cunha LA, Yui KC, Huhtala MF, Barcellos DC, Prakki A et al. Influence of Nd:YAG laser on the bond strength of self-etching and conventional adhesive systems to dental hard tissues. Oper Dent. 2013; 38(4):447-55.

23. Arslan S, Ertaş H, Zorba YO. Effect of a plant-based hemostatic agent on microleakage of self-etching adhesives. Med Oral Patol Oral Cir Bucal. 2013; 18(1):e124-9.

24. Nowicka A, Parafiniuk M, Lipski M, Lichota D, Buczkowska-Radlinska J. Pulpo-dentin complex response after direct capping with self-etch adhesive systems. Folia Histochem Cytobiol. 2012; 50(4):565-73.

25. Batra C, Nagpal R, Tyagi SP, Singh UP, Manuja N. In vitro bonding effectiveness of three different one-step self-etch adhesives with additional enamel etching. J Investig Clin Dent. 2014; 5(3):226-36.

26. Pei D, Liu S, Huang C, Du X, Yang H, Wang Y et al.
Effect of pretreatment with calcium-containing desensitizer on the dentine bonding of mild self-etch adhesives. Eur J Oral Sci. 2013; 121 (3 Pt 1):204-10.

27. Simões DM, Basting RT, Amaral FL, Turssi CP, França FM. Influence of chlorhexidine and/or ethanol treatment on bond strength of an etch-and-rinse adhesive to dentin: an in vitro and in situ study. Oper Dent. 2014; 39(1):64-71.

28. Dayem RN, Tameesh MA. A new concept in hybridization: Bromelain enzyme for deproteinizing dentin before application of adhesive system. Contemp Clin Dent. 2013; 4(4):421-6.

29. Perdigão J, Muñoz MA, Sezinando A, Luque-Martinez IV, Staichak R, Reis A et al. Immediate adhesive properties to dentin and enamel of a universal adhesive associated with a hydrophobic resin coat. Oper Dent. 2014; 39(5):489-99.

30. Malekipour MR, Razavi SM, Khazaei S, Kazemi S, Behnamanesh M, Shirani F. Histologic Evaluation of Human Pulp Response to Total Etch and Self Etch Adhesive Systems. Iran Red Crescent Med J. 2013; 15(5):428-31.

31. Thanatvarakorn O, Nakajima M, Prasansuttiporn T, Ichinose S, Foxton RM, Tagami J. Effect of smear layer deproteinizing on resin-dentine interface with self-etch adhesive. J Dent. 2014; 42(3):298-304.

32. Marchesi G, Frassetto A, Mazzoni A, Apolonio F, Diolosà $\mathrm{M}$, Cadenaro $\mathrm{M}$ et al. Adhesive performance of a multi-mode adhesive system: 1-Year in vitro study. J Dent. 2014; 42(5):603-12.

33. Muñoz MA, Sezinando A, Luque-Martinez I, Szesz AL, Reis A, Loguercio AD et al. Influence of a hydrophobic resin coating on the bonding efficacy of three universal adhesives. J Dent. 2014; 42(5):595-602.

34. Loguercio AD, Luque-Martinez I, Muñoz MA, Szesz AL, Cuadros-Sánchez J, Reis A. A Comprehensive laboratory screening of three-step etch-and- rinse adhesives. Oper Dent. 2014; 39(6):652-62.

35. Mirmohammadi H, Khosravi K, Kashani K, Kleverlaan CJ, Feilzes AJ. Influence of filler existence on microleakage of a self-etch adhesive system. J Conserv Dent. 2014; 17(2):175-8

36. Wagner A, Wendler M, Petschelt A, Belli R. Bonding performance of universal adhesives in different etching modes. J Dent. 2014; 42(7):800-7.

37. Rodriguez-Priego ME, Bolaños-Carmona MV, González-Lópes S, Rodriguez-Navarro A, SanchézSanchéz MP. Ferric Sulphate alterations on Primary dentin and the adhesive interface. J Adhes Dent. 2014; 16(4):347-56.

38. Elkassas DW, Fawzi EM, Zohairy A. The effect of cavity disinfectants on the micro-shear bond strength of dentin adhesives. Eur J Dent. 2014; 8(2):184-90.

39. Moghaddas MJ, Moosavi H, Ghavamnasiri M. Microleakage evaluation of adhesive systems following pulp chamber irrigation with sodium hypochlorite. J Dent Res Dent Clin Dent Prospects. 2014; 8(1):21-6.

40. Aman N, KhanFR , Salim A, Farid H. A randomized control clinical trial of fissure sealant retention: Self etch adhesive versus total etch adhesive. J Conserv Dent. 2015; 18(1):20-4.

41. Almahdy A, et al. An MMP-inhibitor modified adhesive primer enhances bond durability to carious dentin. Dent Mater. 2015 May;31(5):594-602.

42. Vinagre A, Ramos J, Messias A, Marques F, Caramelo 
F, Mata A. Microtensile bond strength and micromorphology os Bur-cut Enamel using five adhesive systems. J Adhes Dent. 2015; 17(2):107-16.

43. Tranquilin JB, Bridi EC, Amaral FL, França FM, Turssi $\mathrm{CP}$, Basting RT. TiF4 improves microtensile bond strength to dentin when using an adhesive system regardless of primer/bond application timing and method. Clin Oral Investig. 2016; 20(1):101-8.

44. Amsler F, Peutzfeldt A, Lussi A, Flury S. Bond strengh of resin composite to dentin with diferente adhesive systems: influence os relative humidity and application time. J Adhes Dent. 2015; 17(3):249-56.

45. Mithiborwala SH, Chaugule V, Katge F, Poojari M, Pujari P, Pammi T. A comparative evaluation of the efficacy of etching by the total etch and self-etch dentin bonding systems in the primary teeth: an in vitro study. Int J Clinical Pediatr Dent. 2015; 8(1):30-6.

46. Alves GC, Sobral APV. Evaluation of biocompatibility of an etch-and-rinse adhesive system based in tertiary butanol applied in deep cavity. Open Dent J. 2015; 9:168-73.

47. Sezinando A, Luque-Martinez I, Muñoz MA, Reis A, Loguercio AD, Perdigão J. Influence of a hydrophobic resin coating on the immediate and 6-month dentin bonding of three universal adhesives. Dent Mater. 2015; 31(10):e236-46.

48. Sharafeddin F, Farhadpour H. Evaluation of shear bond strength of total- and self-etching adhesive systems after application of chlorhexidine to dentin contaminated with a hemostatic agent. J Dent (Shiraz). 2015; 16(3):175-81.

49. Chauhan K, Basavanna RS, Shivanna V. Effect of bromelain enzyme for dentin deproteinization on bond strength of adhesive system. J Conserv Dent. 2015; 18(5):360-3.

50. Montagner AF, Skupien JA, Borges MF, Krejci I, Bortolloto T, Susin A. Effect of sodium hypochlorite as dentinal pretreatment on bonding strength of adhesive systems. Indian J Dent Res. 2015; 26(4):416-20.

51. Carvalho C, Fernandes FP, Freitas VP, França FMG, Basting RT, Turssi CP et al. Effect of green tea extract on bonding durability of an etch-and-rinse adhesive system to cariesaffected dentin. J Appl Oral Sci, 2016; 24(3):211-7.

52. Takada M, Suzuki M, Tsujimura MH, Shinkai K. Effect of adhesive system application for cavities prepared with erbium, chromium: yttrium scandium gallium garnet laser on rat dental pulp tissue. Odontology. 2017; 105:300-10.

\section{CONFLITO DE INTERESSES}

Os autores declaram não haver conflitos de interesse.

\section{AUTOR PARA CORRESPONDÊNCIA}

Paulo Ladislau Saviczki

paulo_s92@hotmail.com

Submetido em 12/09/2017

Aceito em 24/10/2017 